\title{
Mossbauer Investigations in Hematite Nanoparticles
}

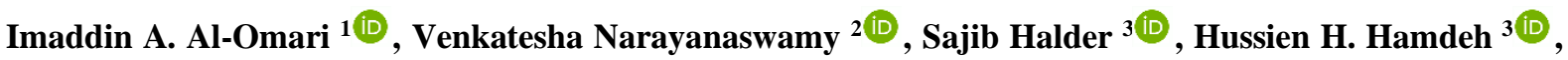 \\ Sulaiman Alaabed ${ }^{2(\mathbb{D})}$, Aleksandr. S. Kamzin ${ }^{4(\mathbb{D}}$, Chandu V. V. Muralee Gopi ${ }^{5(\mathbb{D})}$, Abbas Khaleel ${ }^{6(\mathbb{D})}$, \\ Bashar Issa $^{7 \text { (D) } \text {, Ihab M. Obaidat }}{ }^{8 *}$ *iD \\ 1 Department of Physics, Sultan Qaboos University, P.O. Box 36, Muscat PC 123, Sultanate of Oman; \\ ialomari@squ.edu.om; \\ 2 Department of Geology, United Arab Emirates University, Al-Ain 15551, UAE; venkateshnrn@ gmail.com; \\ 3 Department of Physics, Wichita State University, Wichita, Kansas 67260, USA; sxhalder@shockers.wichita.edu; \\ hussein.hamdeh@wichita.edu; \\ 4 Ioffe Physical-Technical Institute, St. Petersburg, 194021, Russia; ASKAM@mail.ioffe.ru; \\ 5 Department of Electrical Engineering, University of Sharjah, Sharjah, P.O. Box 27272, UAE; naga5673@gmail.com; \\ 6 Department of Chemistry, United Arab Emirates University, Al-Ain 15551, UAE; abbask@uaeu.ac.ae; \\ 7 Department of Medical Diagnostic Imaging, College of Health Sciences, University of Sharjah, Sharjah, P.O. Box 27272, \\ UAE; bissa@sharjah.ac.ae; \\ 8 Department of Physics, United Arab Emirates University, Al-Ain 15551, UAE; iobaidat@uaeu.ac.ae; \\ * Correspondence: iobaidat@uaeu.ac.ae (I.M.O.);
}

Scopus Author ID 10440642200

Received: 2.08.2021; Revised: 15.09.2021; Accepted: 20.09.2021; Published: 16.10.2021

\begin{abstract}
Hematite nanoparticles of average size $20 \mathrm{~nm}$ were synthesized using the sol-gel method, and the structural characterizations were conducted using XRD and TEM. The XRD profile revealed a small fraction of the maghemite phase and the main hematite phase. Mössbauer spectroscopy was used to study the magnetic structure of the particles and revealed a third but very slight non-magnetic phase. Mössbauer spectrum shows 35\% of the nanoparticles exhibiting superparamagnetism. The weighted average Morin transition temperature for the particles determined by Mössbauer is $262 \mathrm{~K}$, which is remarkably similar to the bulk value and higher than the Morin transition determined by VSM (about $250 \mathrm{~K})$. The reported findings on the hematite nanoparticles will help understand the enhanced ferromagnetic behavior of the hematite nanoparticles at room temperature, which is crucial for potential applications.
\end{abstract}

Keywords: nanoparticles; hematite; Morin transition; surface spins.

(C) 2021 by the authors. This article is an open-access article distributed under the terms and conditions of the Creative Commons Attribution (CC BY) license (https://creativecommons.org/licenses/by/4.0/).

\section{Introduction}

Nanoparticles have stimulated the interest of researchers in recent years, and they have been thoroughly investigated due to their numerous potential in biotechnology, environmental protection, data storage, magnetic sensors, and drug delivery [1,2]. The properties of nanoparticles are determined by the particles' size, shape, morphology, crystallinity, surface effects, and inter-particle interactions [3,4]. As a result, a thorough understanding of nanoparticles' fundamental properties is required before they can be widely used. Iron oxide nanoparticles have drawn remarkable attention in biomedical applications like MRI contrast agents and magnetic hyperthermia [5,6]. Iron oxide exists mainly in the form of maghemite, magnetite, and hematite. Maghemite and magnetite have a spinel cubic structure, where Fe spins are distributed in tetrahedral and octahedral sites, which are magnetically coupled through 
the double exchange. Both phases are ferrimagnetic due to the opposite spins of A (tetrahedral) and B (octahedral) sites [7].

The hematite is the most stable oxide of iron commonly found in rocks and soil naturally. Because of its stability and low cost of mining, hematite is the most studied natural material for catalytic, gas sensors, electrochemical, inorganic pigments, and water purification applications [8,9]. Hematite is synthesized by various chemical and physical methods. Liu et al. were able to synthesize quasi, irregular cubic, parallel hexahedron sphere, nanosheet, and hexagon-shaped $\alpha-\mathrm{Fe}_{2} \mathrm{O}_{3}$ structures using anions surfactants like $\mathrm{OH}^{-}, \mathrm{Cl}^{-}, \mathrm{SO}_{4}{ }^{2-}, \mathrm{NO}^{3-}$ and $\mathrm{CH}_{3} \mathrm{COOH}$. They have shown that the magnetic and optical properties of $\alpha-\mathrm{Fe}_{2} \mathrm{O}_{3}$ nanoparticles significantly depend on the shape of the nanoparticles [10]. At $\mathrm{T}_{\mathrm{M}} 263 \mathrm{~K}$, a spin-reorientation phenomena (magnetic phase transition) occurs in antiferromagnetic crystalline (rhombohedral) hematite $\left(\alpha-\mathrm{Fe}_{2} \mathrm{O}_{3}\right)$, which is known as the Morin transition $\mathrm{T}_{M}$ [11]. The Morin transition is caused by the competition between two anisotropies of similar magnitude but opposite signs. The material is an antiferromagnet when the two magnetic sublattices are antiparallel and oriented toward the rhombohedral axis [111] below TM. The moments above $\mathrm{T}_{\mathrm{M}}$ are on the basal plane (111), with some canting from the antiferromagnetic axis, due to the superexchange interaction [12]. As a result of the low net magnetization, the material is only weakly ferromagnetic. The Morin temperature lowers as particle size decreases and tends to zero for particles with a diameter of $10-20 \mathrm{~nm}$ or less [13-16]. In calculating the $\mathrm{T}_{\mathrm{M}}$ value, the surface impact plays a vital influence. At various stages, $\alpha-\mathrm{Fe}_{2} \mathrm{O}_{3}$ nanoparticles exhibit superparamagnetic, weak-ferromagnetic, and antiferromagnetic properties, making them intriguing materials for fundamental research [17].

We have reported Morin transition for the $\alpha-\mathrm{Fe}_{2} \mathrm{O}_{3}$ nanoparticles with respect to various cooling fields used for obtaining magnetization with respect to temperature. It was found that the Morin transition temperature is slightly field-dependent, and it occurred around $250 \mathrm{~K}$ [18]. The extent of the transition was broad, and it was attributed to the wide distribution of sizes. The particles also possessed non-zero magnetization in the antiferromagnetic state and they possess significant magnetization at room temperature. Attaining considerable ferromagnetic nature for hematite nanoparticles at room temperature is of paramount importance for the potential applications.

Mössbauer spectroscopy measurements have been conducted to understand the magnetic and structural properties of the hematite $\left(\alpha-\mathrm{Fe}_{2} \mathrm{O}_{3}\right)$ nanoparticles at different temperatures were starting from $300 \mathrm{~K}$ down to $140 \mathrm{~K}$. The Mössbauer measurement accurately detected minute fluctuations of nuclear energy levels induced by interactions of the resonant nucleus with its surrounding electronic system through hyperfine interactions [19]. Morin transition temperature obtained from Mossbauer spectroscopy was compared with that obtained from the magnetic measurements [18]. We have indicated the ferrimagnetic (Maghemite) phase as ferromagnetic because of the similar response to the magnetic field.

\section{Materials and Methods}

\subsection{Synthesis.}

Hematite $\left(\alpha-\mathrm{Fe}_{2} \mathrm{O}_{3}\right)$ nanoparticles were synthesized by dissolving $\mathrm{Fe}\left(\mathrm{NO}_{3}\right)_{3} .9 \mathrm{H}_{2} \mathrm{O}$ salt in 2-propanol solvent and propylene oxide promotor, which acts as condensing agent. In the synthesis method, $10.7 \mathrm{~g}$ of iron salt was dissolved in $100 \mathrm{ml}$ of 2-propanol and $25 \mathrm{ml}$ propylene oxide mixture under constant stirring for 4 hours. Upon continuous stirring, the solution turns 
into reddish-brown colloidal, and it is further aged for 24 hours, followed by evaporation of the solvent at $80{ }^{\circ} \mathrm{C}$ in a water bath. The brown precipitate is further washed with water and dried for 1 hour at $120{ }^{\circ} \mathrm{C}$. Finally, the solid obtained is calcined at 350 and $500{ }^{\circ} \mathrm{C}$ for 1 and 4 hours, respectively.

\subsection{Structural characterization.}

The structure and morphology of the nanoparticles were determined by X-ray diffraction (XRD) and transmission electron microscopy (TEM) images. XRD characterization was carried out using a Shimadzu-6100 powder XRD diffractometer with $\mathrm{Cu}$-Ka radiation with wavelength $1.542 \AA$. Diffraction data were obtained at an angle range of 20-80 degrees with a $1 \mathrm{deg} /$ minute scan rate. TEM images were obtained using the Philips electron microscope of CM10. The average crystalline size is calculated using the Scherrer formula from the full width half maximum (FWHM) of the highest intensity peak (110).

\subsection{Mössbauer measurements.}

Mössbauer spectroscopy was used to investigate the microscopic details of Morin transition, particle size, and magnetic behavior about the sample. To record the Mössbauer spectra, a conventional constant acceleration spectrometer (MS-1200, Ranger Scientific) was utilized in transmission mode. For this experiment, a radioactive ${ }^{57} \mathrm{Co}$ embedded in the $\mathrm{Rh}$ matrix was used as a radioactive source. The spectra were recorded using a gas detector.The sample was investigated at a cryogenic temperature in the -10 to $+10 \mathrm{~mm} / \mathrm{s}$ velocity range. A vibration-free closed-cycle refrigerator was used to store the sample. The cryostat was used to cool the sample and take the measurements at different temperatures. All the isomer shifts were measured relative to the centroid of $\alpha$-iron.

\section{Results and Discussion}

The XRD profile obtained from the sol-gel-synthesized hematite nanoparticles is shown in Figure 1. The diffraction pattern consists of peaks corresponding to $\alpha-\mathrm{Fe}_{2} \mathrm{O}_{3}$ and a small fraction of the maghemite phase. Though it indicates the formation of mixed-phase, the fraction of maghemite is very low, which is evident from the intensity ratio of maghemite and hematite peaks. Using POWDER-CELL, the unit cell parameters were determined. The lattice dimensions are $\mathrm{a}=5.0339 \AA$ and $\mathrm{c}=13.7866 \AA$ with a cell volume of $302.5545(\AA)^{3}$. The XRD pattern obtained is in agreement with the expected XRD profile of the $\alpha-\mathrm{Fe}_{2} \mathrm{O}_{3}$ phase. By using FWHM of the highest intensity peak (110) corresponding to the $\alpha-\mathrm{Fe}_{2} \mathrm{O}_{3}$ phase, the average crystallite size of the nanoparticles was obtained. From the XRD profile and using the Scherrer formula, the mean diameter of the nanoparticles was calculated to be about $20 \mathrm{~nm}$. The nanoparticles' bright-field TEM image is shown in Figure 2. The nanoparticles have a twisted spherical appearance and are broadly distributed in size. The size distribution histogram is obtained by measuring the sizes of the individual nanoparticles using sigma scan software, and the histogram is shown in Figure 2a. The average sizes of the nanoparticles are $17 \mathrm{~nm}$, and the size distribution is broad, with a significant number of particles having sizes of 11-13 nm. 


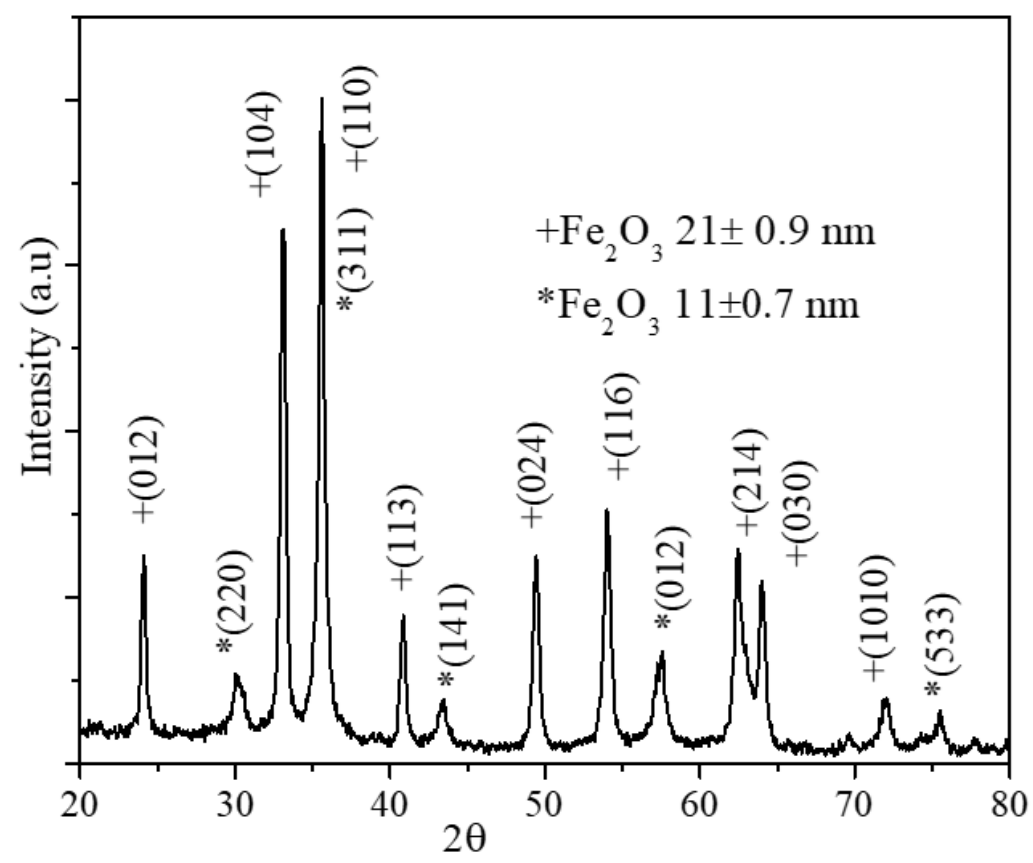

Figure 1. X-ray diffraction pattern of hematite nanoparticles. (This Figure was reported in our earlier work [18].
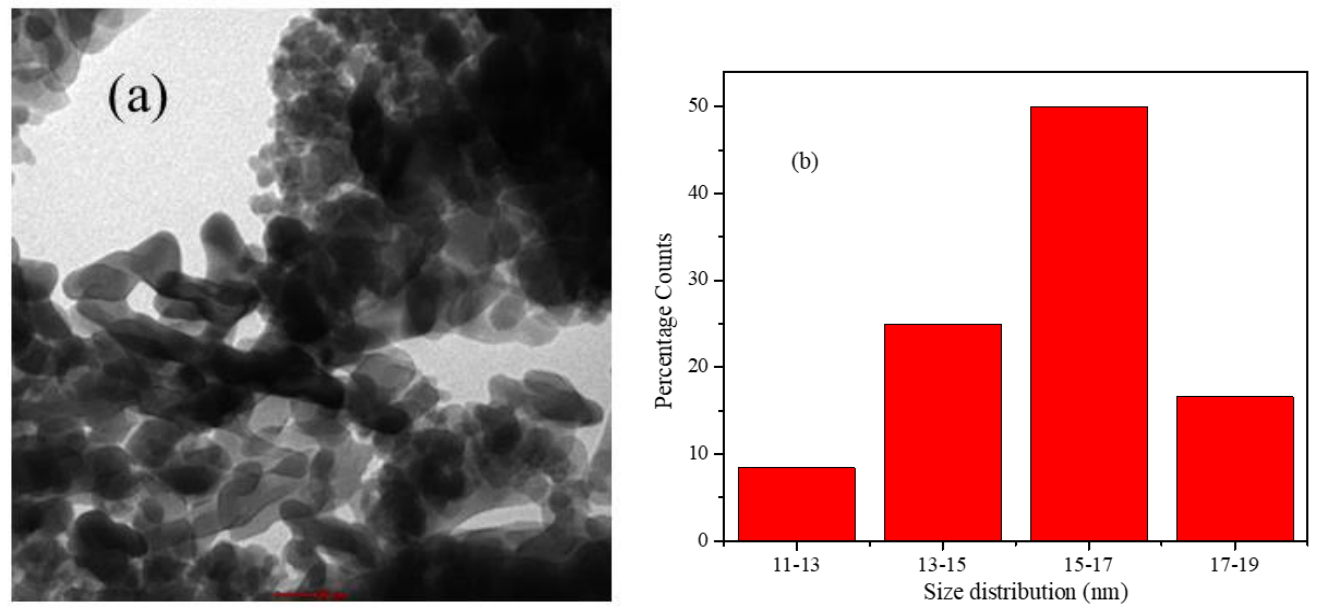

Figure 2. (a) TEM bright-field images of hematite nanoparticles illustrating the characteristic particle morphologies. (b) Size distributions of the nanoparticles.

We used Mössbauer spectroscopy to investigate the Morin transition over a large temperature range and the magnetic behavior at each temperature. Because the Mössbauer spectrum is derived from distinct Fe atom positions in the sample, it can be used to calculate the precise fraction of Fe-based phases. Mossbauer additionally provides reliable spin dynamics information on a time range of $10^{-7}$ to $10^{-9} \mathrm{~s}(\mathrm{c})$. For paramagnetic Fe atoms, a Mössbauer spectrum normally shows a single or two resonance lines (doublet) or six (sextet) resonance lines for magnetically arranged Fe atoms. Isomer shift (IS), quadrupole splitting (QS), and hyperfine magnetic splitting are the three primary hyperfine properties found in each spectrum (HMF). The isomer shift reveals information about the Fe ion's valence and spin states. The quadrupole splitting method, which is dependent on Fe site symmetry, also provides information on the valence and spin states[20-23]. The hyperfine magnetic field offers magnetic information about the materials. In this study, the Mössbauer data were analyzed by the least-squares peak fitting method. This fitting method gave all the magnetic fractions, hyperfine magnetic field, quadrupole splitting, and isomer shift values with their uncertainties. 
Measured Mössbauer spectra at different temperatures are shown in Figure 3. Figure 3 shows the experimental data as dots and the least-squares fit as solid lines. Table 1 summarizes the sub-spectrum with their percentages, as well as hyperfine metrics such as isomer shift (IS), quadrupole splitting (QS), and hyperfine magnetic field (HMF). Figure 3 shows that at ambient temperature, the Mössbauer spectrum has two components: one is a conventional magnetic sextet (sextet 1), while the other is a collapsed sextet. The HMF for component 1 (sextet 1) at ambient temperature is $521 \mathrm{kG}$, which is slightly higher than the HMF for pure hematite $(\alpha-$ $\left.\mathrm{Fe}_{2} \mathrm{O}_{3}\right)(518 \mathrm{kG})$. The nanosized nature of our sample may explain the tiny discrepancy from the literature value. For the first component, the isomer shift value is $0.419 \mathrm{~mm} / \mathrm{s}$. The collapsed sextet lacks both the HMF and the isomer shift. At this temperature, the quadrupole splitting is $-0.188 \mathrm{~mm} / \mathrm{s}$, indicating that our sample is a weakly ferromagnetic material at room temperature. The hematite $\left(\alpha-\mathrm{Fe}_{2} \mathrm{O}_{3}\right)$ nanoparticles are weak ferromagnetic, as indicated by the negative sign. The negative sign indicates that the hematite $\left(\alpha-\mathrm{Fe}_{2} \mathrm{O}_{3}\right)$ nanoparticles are weak ferromagnetic. In addition, Mössbauer spectra of the sample collected below the room temperatures starting from $285 \mathrm{~K}$ to $140 \mathrm{~K}$ were recorded, and a few representative spectra are shown in Figure 3. Table 1 contains the hyperfine parameters. At any temperature between 285 and $240 \mathrm{~K}$, the Mössbauer spectrum shows two separate sextets and a doublet. Because there is no non-magnetic component (collapsed sextet), all particles are magnetic at these temperatures, with a little amount of paramagnetic (5\%). (doublet).

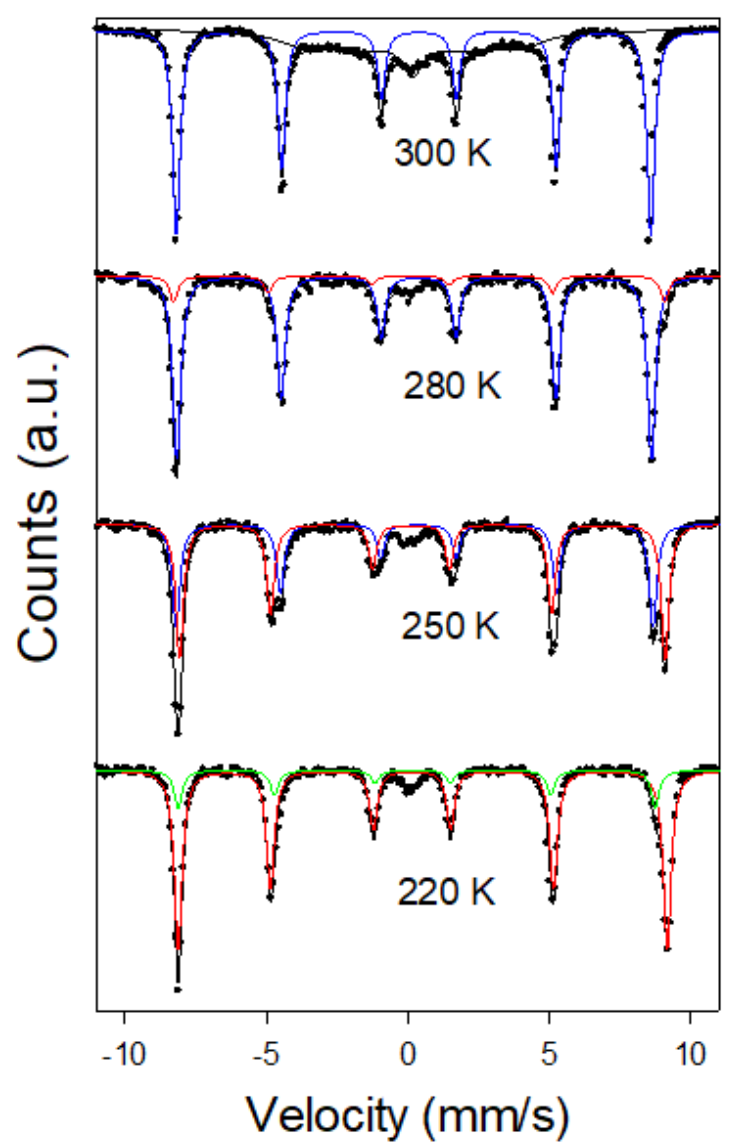

Figure 3. Representative Mössbauer spectra at temperatures 220, 250, 280, and $300 \mathrm{~K}$ (Black superparamagnetic; Blue weak-ferromagnetic; Red antiferromagnetic; Green Maghemite).

The blue color in the Mössbauer spectrum represents sextet 1, while the red color is sextet 2 . Both the sextets are in good magnetic order. The doublet at each temperature (around 5 percent) indicates the presence of tiny crystallite sizes of the particles (with a diameter of $\approx 5$ 
$\mathrm{nm}$ or less). The appearance of sextet 2 at $285 \mathrm{~K}$, which was absent at $300 \mathrm{~K}$, indicates that some of the particles have transitioned from weakly ferromagnetic to antiferromagnetic states. The phase transition, sometimes known as the Morin transition, is the name given to this transition. At $285 \mathrm{~K}, 87$ percent of particles are weakly ferromagnetic (sextet 1), and just 7\% are antiferromagnetic (sextet 2). (sextet 2). Antiferromagnetic is the proportion that went through phase transition ( 7 percent at $285 \mathrm{~K})$. We can identify hematite $\left(\alpha-\mathrm{Fe}_{2} \mathrm{O}_{3}\right)$ nanoparticles in a weakly ferromagnetic state that did not undergo Morin transition and particles in an antiferromagnetic state that did undergo Morin transition simultaneously. So, depending on the temperature, size, and chemical structure of the materials, we identified a variable fraction of two distinct hematite $\left(\alpha-\mathrm{Fe}_{2} \mathrm{O}_{3}\right)$ components between $285 \mathrm{~K}$ and $240 \mathrm{~K}$. At each temperature, the QS value reveals the proportions of ferromagnetic and antiferromagnetic materials in the sample. The QS values (in mm/s) for sextet 1 at temperatures of $285 \mathrm{~K}, 280 \mathrm{~K}, 275 \mathrm{~K}, 270 \mathrm{~K}$, and $265 \mathrm{~K}$ are $-0.188,-0.159,-0.18,-0.164$, and -0.145 , respectively, with the QS values (in $\mathrm{mm} / \mathrm{s}$ ) gradually dropping with decreasing temperature. This means that when the temperature drops, the ferromagnetic properties gradually weaken. QS (in $\mathrm{mm} / \mathrm{s}$ ) for sextet 2 are 0.345 , $0.355,0.398,0.948,0.405$ at $285 \mathrm{~K}, 280 \mathrm{~K}, 275 \mathrm{~K}, 270 \mathrm{~K}$, and $265 \mathrm{~K}$, respectively. As can be observed in Figure 4, this shows an increasing pattern, showing that a considerable fraction of the hematite $\left(\alpha-\mathrm{Fe}_{2} \mathrm{O}_{3}\right)$ nanoparticles undergo Morin transition at each temperature, eventually becoming stronger antiferromagnetic.

The coupling between neighboring atoms becomes negative as the temperature of the sample drops, and the materials become antiferromagnetic. For sextet 1 (weakly ferromagnetic), the QS versus temperature increases over the whole temperature range. The Qs for sextet 2 (antiferromagnetic) differs from that of sextet 1, in which the QS value grew at first and then remained constant at lower temperatures. The blue color (sextet 1) representing ferromagnetic in the Mössbauer spectrum at $285 \mathrm{~K}$, which has the largest area (87 percent); at $280 \mathrm{~K}$, the area becomes smaller (83 percent), and it continues to decrease at lower temperatures and eventually became 25 percent at $240 \mathrm{~K}$. The red color (sextet 2), which represented antiferromagnetically and exhibited a tiny peak at $285 \mathrm{~K}$ ( 7 percent), grew larger and greater at following temperatures, reaching 70 percent at $240 \mathrm{~K}$. As the temperature was lowered, more and more materials passed through the Morin transition, and the fraction rose as the temperature was lowered. When materials undergo Morin transition, structural deformation occurs, which is particularly sensitive to the QS. Since the phase transition influences isomer shift, the materials changing from ferromagnetic to antiferromagnetic state changes isomer shift. Since there are two different iron components (sextet 1 and sextet 2) at each temperature, each temperature has two different isomer shifts. We found that the average isomer shift is increasing with decreasing the temperature, which is due to the second-order Doppler shift. It is observed that as the temperature was reduced, the isomer shift for antiferromagnetic materials increased. The HMF is derived mostly from the magnetic moments of iron. All the HMF in iron oxide materials (hematite, maghemite, magnetite) are so close to each other that we can't always tell them apart in mixed iron phases materials. Table 1 shows the temperature dependency of the HMF for sextet 1 and sextet 2. The HMF gradually increases as the temperature is reduced, as seen in the table. The average HMF is seen to increase when the temperature drops. The materials are in good magnetic order at temperatures ranging from 285 $\mathrm{K}$ to $240 \mathrm{~K}$, and they are in a combination of weakly ferromagnetic and antiferromagnetic states. The results obtained at $220 \mathrm{~K}$ and $180 \mathrm{~K}$ significantly differed. We have two sextets at these temperatures. Sextet 1 is made up of antiferromagnetic maghemite $\left(\gamma-\mathrm{Fe}_{2} \mathrm{O}_{3}\right)$. Hematite 
$\left(\alpha-\mathrm{Fe}_{2} \mathrm{O}_{3}\right)$ is the source of Sextet 2, which is also antiferromagnetic. Both sextets have a positive QS, albeit sextet 2 has a greater QS than sextet 1. Both sextets have great magnetic order and are magnetic. At these temperatures, the magnetic state is slightly antiferromagnetic. The presence of maghemite $\left(\gamma-\mathrm{Fe}_{2} \mathrm{O}_{3}\right)$ and hematite $\left(\alpha-\mathrm{Fe}_{2} \mathrm{O}_{3}\right)$ at $220 \mathrm{~K}$ and $180 \mathrm{~K}$ confirms that the sample has a mixed-phase and so coincides with the X-ray diffraction data. The lower amount of maghemite $\left(\gamma-\mathrm{Fe}_{2} \mathrm{O}_{3}\right)$ in the sample (16 and 12 percent at 220 and 180 degrees Celsius, respectively) indicates that hematite $\left(\alpha-\mathrm{Fe}_{2} \mathrm{O}_{3}\right)$ is the dominating phase. At $140 \mathrm{~K}$ to $20 \mathrm{~K}$, Mössbauer's measurements revealed only the existence of sextet 2 and no evidence of sextet 1. In the Mössbauer spectrum, the red color (sextet 2) occupies the largest area, whereas the blue color (sextet 1$)$ has vanished completely. Hematite $\left(\alpha-\mathrm{Fe}_{2} \mathrm{O}_{3}\right)$ contributes to the positive QS. With a drop in temperature, both HMF and isomer shifts increased. At these temperatures, the Morin transition is 100 percent, which implies that all particles have entered phase transition and are significantly antiferromagnetic.

Table 1. Fits of the Mössbauer spectra yielded the following results. The table shows the parameters retrieved from the fits in Figure 10 for each of the doublets and sextets that were fit at each temperature. HMF is the Hyperfine Magnetic Field and in all fits have an uncertainty of $\pm 5 \mathrm{kG}$. QS Is the Quadrupole Split with an uncertainty of $\pm 0.05 \mathrm{~mm} / \mathrm{s}$ on each measurement. The Isomer Shifts (IS) also have $\pm 0.05 \mathrm{~mm} / \mathrm{s}$ uncertainty.

The iron fractions $(\mathrm{Fe} \%)$ have been extracted from the areas of the components of the fit with an uncertainty of $\pm 0.03= \pm 3 \%$. Antiferromagnetic hematite (AFM-H, sextet 2 ) was uniquely identified from the large positive

QS, the percentage of the hematite that underwent Morin Transition is indicated as (HUMT).

\begin{tabular}{|c|c|c|c|c|c|c|}
\hline $\mathbf{T}(\mathbf{K})$ & sub-spectrum & HMF (kG) & $\mathbf{Q S}(\mathbf{m m} / \mathbf{s})$ & IS $(\mathbf{m m} / \mathbf{s})$ & $\mathrm{Fe} \%$ & Phase \\
\hline \multirow[t]{2}{*}{300} & sextet 1 & 521 & -0.19 & 0.42 & 65 & mainly hematite \\
\hline & collapsed sextet & & & & 35 & \\
\hline \multirow[t]{3}{*}{285} & sextet 1 & 521 & -0.19 & 0.42 & 87 & mainly hematite \\
\hline & sextet $2(\mathrm{AFM}-\mathrm{H})$ & 539 & 0.35 & 0.31 & 7 & HUMT \\
\hline & doublet & & 0.15 & 0.05 & 6 & \\
\hline \multirow[t]{3}{*}{280} & sextet 1 & 521 & -0.16 & 0.42 & 83 & mainly hematite \\
\hline & sextet $2($ AFM-H $)$ & 539 & 0.36 & 0.31 & 11 & HUMT \\
\hline & doublet & & 0 & 0.02 & 6 & \\
\hline \multirow[t]{3}{*}{275} & sextet 1 & 523 & -0.18 & 0.41 & 71 & mainly hematite \\
\hline & sextet $2($ AFM-H $)$ & 532 & 0.4 & 0.41 & 24 & HUMT \\
\hline & doublet & & 0 & 0.09 & 5 & \\
\hline \multirow[t]{3}{*}{270} & sextet 1 & 523 & -0.16 & 0.41 & 57 & mainly hematite \\
\hline & sextet $2(\mathrm{AFM}-\mathrm{H})$ & 533 & 0.39 & 0.41 & 39 & HUMT \\
\hline & doublet & & 0.44 & 0.11 & 4 & \\
\hline \multirow[t]{3}{*}{265} & sextet 1 & 524 & -0.15 & 0.41 & 55 & mainly hematite \\
\hline & sextet $2(\mathrm{AFM}-\mathrm{H})$ & 534 & 0.41 & 0.42 & 40 & HUMT \\
\hline & doublet & & 0 & 0.02 & 5 & \\
\hline \multirow[t]{3}{*}{260} & sextet 1 & 523 & -0.16 & 0.42 & 56 & mainly hematite \\
\hline & sextet $2(\mathrm{AFM}-\mathrm{H})$ & 534 & 0.40 & 0.43 & 38 & HUMT \\
\hline & doublet & & 0 & 0.12 & 6 & \\
\hline \multirow[t]{3}{*}{250} & sextet 1 & 524 & -0.14 & 0.42 & 41 & hematite \\
\hline & sextet $2(\mathrm{AFM}-\mathrm{H})$ & 533 & 0.40 & 0.43 & 54 & HUMT \\
\hline & doublet & & 0.40 & 0.08 & 5 & \\
\hline \multirow[t]{3}{*}{240} & sextet 1 & 524 & -0.06 & 0.40 & 25 & hematite \\
\hline & sextet $2(\mathrm{AFM}-\mathrm{H})$ & 535 & 0.41 & 0.44 & 70 & HUMT \\
\hline & doublet & & 0.45 & 0.12 & 5 & \\
\hline \multirow[t]{3}{*}{220} & sextet 1 & 524 & 0.15 & 0.35 & 16 & Maghemite \\
\hline & sextet $2($ AFM-H $))$ & 537 & 0.38 & 0.45 & 79 & HUMT \\
\hline & doublet & & 0.31 & 0.10 & 5 & \\
\hline \multirow[t]{3}{*}{180} & sextet 1 & 527 & 0.17 & 0.28 & 12 & Maghemite \\
\hline & sextet $2(\mathrm{AFM}-\mathrm{H})$ & 540 & 0.39 & 0.50 & 83 & HUMT \\
\hline & doublet & & 0 & 0.13 & 5 & \\
\hline
\end{tabular}

IV (d). Average Morin transition temperature.

We used a temperature range of $180 \mathrm{~K}$ to $285 \mathrm{~K}$ to obtain the average Morin transition temperature $\mathrm{TM}>$ for our sample. We started by determining bin width (the temperature 
difference between two temperatures) and bin-center (the center of two temperatures). The average Morin transition temperature may then be calculated from the data using the following equation 4:

$$
<T_{M}>=\frac{\sum_{i=1}^{N} \frac{T_{i}+T_{i+1}}{2} \cdot \frac{f_{i+1}-f_{i}}{T_{i}-T_{i+1}}}{\sum_{i=1}^{N} \frac{f_{i+1}-f_{i}}{T_{i}-T_{i+1}}}
$$

where $\mathrm{Ti}$ is the temperature of measurement $\mathrm{i}$, fi is the fraction that has transitioned at temperature $\mathrm{Ti}$, and the summation is over the $\mathrm{N}$ temperatures spanning the range of the transition, in this case from $220 \mathrm{~K}$ to $285 \mathrm{~K}$, although including a wider range will not change the result as additional terms in the sums of both numerator and denominator vanish when fi+1 - $\mathrm{fi}=0$ as will be the case when the fraction is constant at 0 or $100 \%$ outside the range of the transition. The result obtained from our calculation is the average Morin transition temperature $\left\langle\mathrm{T}_{\mathrm{M}}\right\rangle=(265 \mathrm{~K} \pm 2) \mathrm{K}$.

The Morin temperature decreases with decreasing the particle size and tends to zero for the particles with size $10-20 \mathrm{~nm}$ or less [24-27]. Surprisingly, the Morin temperature is very similar to the Morin temperature for the bulk material. In Ref.[28], it was suggested that the Morin transition occurs because of a competition between two anisotropy terms of comparable magnitude and opposite signs. These are the local ionic anisotropy term, fine structure anisotropy (due to the spin-orbit coupling), and a long-range dipolar anisotropy term. The fine structure anisotropy directs the easy magnetic axis along the c-axis, while the dipolar anisotropy directs the easy magnetic axis within the c-plane. Below $\mathrm{T}_{M}$ the magnitude of the fine structure anisotropy term becomes larger than that of dipolar anisotropy. These two anisotropy terms have different temperature dependences. The competition between the two anisotropy terms is dependent on several factors, including particle size, particle shapes, lattice expansion, defects, and surface effects [29]. Hence, any change in crystalline anisotropy will result in changes in the weights of the competing anisotropy terms and thus will result in a change in $\mathrm{T}_{\mathrm{M}}$.

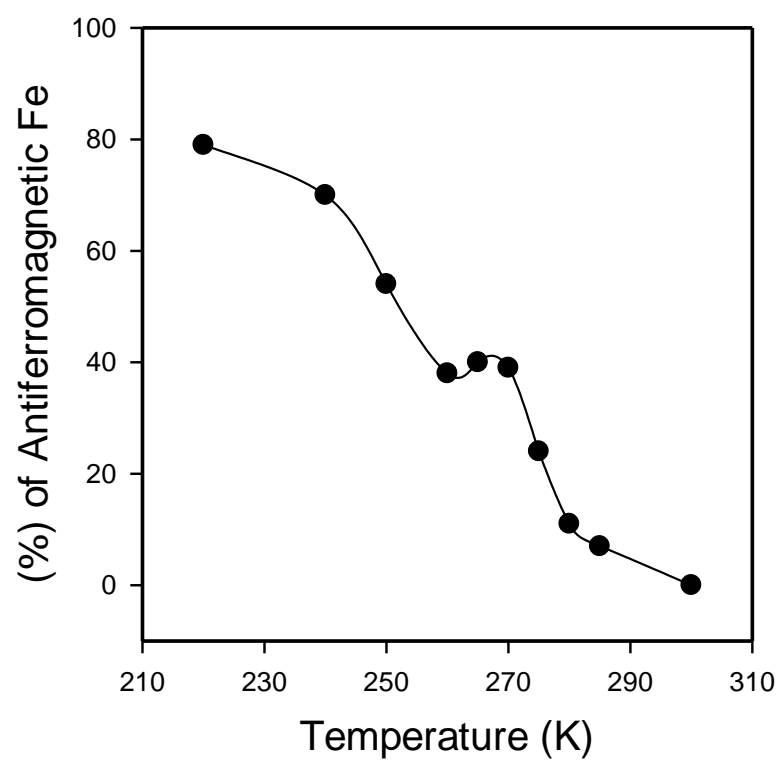

Figure 4. Percentage of antiferromagnetic fraction (sextet 2) as a function of temperature. The plot shows the antiferromagnetic fraction for sextet 2 to increase gradually as we decreased the temperature.

At the surface of the nanoparticles, crystalline anisotropy is expected to change from bulk, leading to changes in $T_{M}$. It was reported that the surface spins locally reduce $T_{M}[30,31]$. 
However, $T_{M}$ in our sample did not display significant suppression regardless of its small size. Hence, there must exist another factor that opposed the suppression size effect. Because our hematite samples contain another magnetic phase, we suggest that the exchange coupling between the two different magnetic phases, mediated by spin-glass regions, suppressed the long-range dipolar anisotropy term and maintained it smaller than the fine structure anisotropy term in a large temperature range.

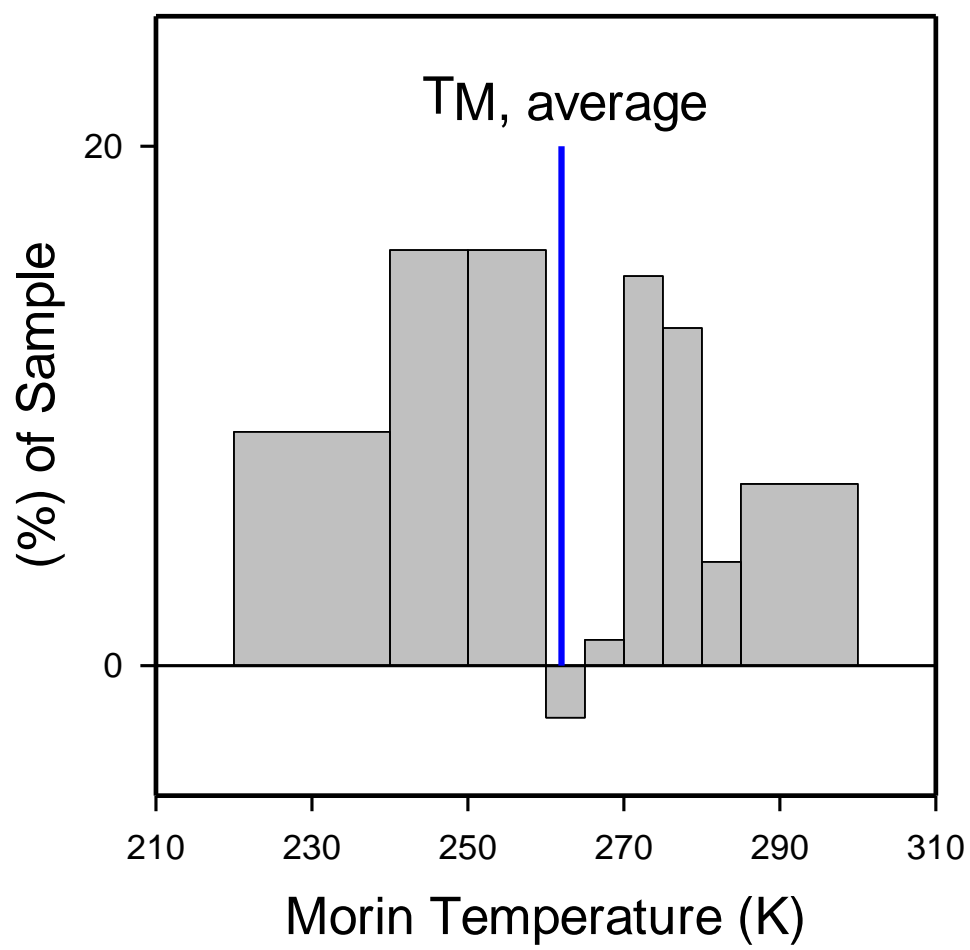

Figure 5. Histogram of the percentage of antiferromagnetic fraction (sextet 2) as a function of Morin temperature. The plot shows the average Morin temperature $\left(\mathrm{T}_{\mathrm{M}}\right.$, average).

Figure 5 shows the percentage of antiferromagnetic fraction as a function of Morin temperature. The percentages of antiferromagnetic fraction were calculated from the area under each sub-spectra. Each area represents the fraction of $\mathrm{Fe}$ in the corresponding phase, as indicated in the table. The percentage of the antiferromagnetic in the histogram is the fraction of the sample turned antiferromagnetic (i.e., underwent Moring transition) between two adjacent measuring temperatures.

\section{Conclusions}

The sol-gel method synthesized hematite nanoparticles were characterized by XRD and TEM. A small fraction of maghemite was observed in the XRD spectrum. The Mössbauer studies at room temperature revealed a third and very slight non-magnetic phase. Mossbauer spectra showed that nanoparticles are in the superparamagnetic state with good magnetic order. The magnetic phase transition started just below room temperature. The percentage of the sample that undergoes Morin transition increases sharply with decreasing temperature and becomes highest at around $140 \mathrm{~K}$. The weighted average Morin transition is found to be (262 $\pm 2) \mathrm{K}$. This value is surprisingly similar to the Morin transition of bulk hematite with large uncertainty. The novel results of Morin transition temperature in our sample were attributed to the indirect magnetic exchange interaction between the AFM and FM nanoparticles mediated by SG regions. 


\section{Funding}

We would like to thank United Arab Emirates University (UAEU) for financially supporting this work under UAEU Program for Advanced Research (UPAR) with Grant no.31S364.

\section{Conflicts of Interest}

The authors declare no conflict of interest. The funders had no role in the study's design, in the collection, analyses, or interpretation of data, in the writing of the manuscript, or in the decision to publish the results.

\section{References}

1. Colombo, M.; Carregal-Romero, S.; Casula, M.F.; Gutiérrez, L.; Morales, M.P.; Böhm, I.B.; Heverhagen, J.T.; Prosperi, D.; Parak, W.J. Biological Applications of Magnetic Nanoparticles. Chem. Soc. Rev. 2012, 41, 4306-4334, https://doi.org/10.1039/C2CS15337H.

2. Ali, A.; Shah, T.; Ullah, R.; Zhou, P.; Guo, M.; Ovais, M.; Tan, Z.; Rui, Y. Review on Recent Progress in Magnetic Nanoparticles: Synthesis, Characterization, and Diverse Applications. Front Chem 2021, 9, 629054, https://doi.org/10.3389/fchem.2021.629054.

3. Abdolrahimi, M.; Vasilakaki, M.; Slimani, S.; Ntallis, N.; Varvaro, G.; Laureti, S.; Meneghini, C.; Trohidou, K.N.; Fiorani, D.; Peddis, D. Magnetism of Nanoparticles: Effect of the Organic Coating. Nanomaterials 2021, 11, 1787, https://doi.org/10.3390/nano11071787.

4. Huang, C.; Chen, X.; Xue, Z.; Wang, T. Effect of Structure: A New Insight into Nanoparticle Assemblies from Inanimate to Animate. Science Advances 2020, 6, eaba1321, https://doi.org/10.1126/sciadv.aba1321.

5. Caspani, S.; Magalhães, R.; Araújo, J.P.; Sousa, C.T. Magnetic Nanomaterials as Contrast Agents for MRI. Materials (Basel) 2020, 13, 2586, https://doi.org/10.3390/ma13112586,

6. Vilas-Boas, V.; Carvalho, F.; Espiña, B. Magnetic Hyperthermia for Cancer Treatment: Main Parameters Affecting the Outcome of In Vitro and In Vivo Studies. Molecules 2020, 25, 2874, https://doi.org/10.3390/molecules25122874.

7. The Iron Oxides: Structure, Properties, Reactions, Occurrences and Uses, 2nd, Completely Revised and Extended Edition | Wiley Available online: (accessed on 9 June 2021).

8. Ashraf, M.; Khan, I.; Usman, M.; Khan, A.; Shah, S.S.; Khan, A.Z.; Saeed, K.; Yaseen, M.; Ehsan, M.F.; Tahir, M.N.; et al. Hematite and Magnetite Nanostructures for Green and Sustainable Energy Harnessing and Environmental Pollution Control: A Review. Chem Res Toxicol 2020, 33, 1292-1311, https://doi.org/10.1021/acs.chemrestox.9b00308.

9. Xue, Y.; Wang, Y. A Review of the $\alpha-\mathrm{Fe}_{2} \mathrm{O}_{3}$ (Hematite) Nanotube Structure: Recent Advances in Synthesis, Characterization, and Applications. Nanoscale 2020, 12, 10912-10932, https://doi.org/10.1039/D0NR02705G.

10. Popov, N.; Bošković, M.; Perović, M.; Zadro, K.; Gilja, V.; Kratofil Krehula, L.; Robić, M.; Marciuš, M.; Ristić, M.; Musić, S.; et al. Effect of Ru ${ }^{3+}$ Ions on the Formation, Structural, Magnetic and Optical Properties of Hematite $\left(\alpha-\mathrm{Fe}_{2} \mathrm{O}_{3}\right)$ Nanorods. Journal of Magnetism and Magnetic Materials 2021, 538, 168316, https://doi.org/10.1016/j.jmmm.2021.168316.

11. Muench, G.J.; Arajs, S.; Matijević, E. The Morin Transition in Small $\alpha-\mathrm{Fe}_{2} \mathrm{O}_{3}$ Particles. physica status solidi(a) 1985, 92, 187-192, https://doi.org/10.1002/pssa.2210920117.

12. Bhowmik, R.N.; Saravanan, A. Surface Magnetism, Morin Transition, and Magnetic Dynamics in Antiferromagnetic $\alpha-\mathrm{Fe}_{2} \mathrm{O}_{3}$ (Hematite) Nanograins. Journal of Applied Physics 2010, 107, 053916, https://doi.org/10.1063/1.3327433.

13. Lee, J.B.; Kim, H.J.; Lužnik, J.; Jelen, A.; Pajić, D.; Wencka, M.; Jagličić, Z.; Meden, A.; Dolinšek, J. Synthesis and Magnetic Properties of Hematite Particles in a "Nanomedusa" Morphology. Journal of Nanomaterials 2014, 2014, e902968, https://doi.org/10.1155/2014/902968.

14. Shimomura, N.; Pati, S.P.; Sato, Y.; Nozaki, T.; Shibata, T.; Mibu, K.; Sahashi, M. Morin Transition Temperature in (0001)-Oriented $\alpha$-Fe2O3 Thin Film and Effect of Ir Doping. Journal of Applied Physics 2015, 117, 17C736, https://doi.org/10.1063/1.4916304. 
15. Seifert, J.; Günzing, D.; Webers, S.; Dulle, M.; Kruteva, M.; Landers, J.; Wende, H.; Schmidt, A.M. Strainand Field-Induced Anisotropy in Hybrid Elastomers with Elongated Filler Nanoparticles. Soft Matter 2021, https://doi.org/10.1039/D0SM02104K.

16. Tsepelev, V.S.; Starodubtsev, Y.N. Nanocrystalline Soft Magnetic Iron-Based Materials from Liquid State to Ready Product. Nanomaterials (Basel) 2021, 11, 108, https://doi.org/10.3390/nano11010108.

17. Roberts, A.P.; Zhao, X.; Heslop, D.; Abrajevitch, A.; Chen, Y.-H.; Hu, P.; Jiang, Z.; Liu, Q.; Pillans, B.J. Hematite $\left(\alpha-\mathrm{Fe}_{2} \mathrm{O}_{3}\right)$ Quantification in Sedimentary Magnetism: Limitations of Existing Proxies and Ways Forward. Geoscience Letters 2020, 7, 8, https://doi.org/10.1186/s40562-020-00157-5.

18. Ihab, M.O.; Sulaiman, A.; Imad, A.A.-O.; Venkatesha, N.; Bashar, I.; Abbas, K. Field-Dependent Morin Transition and Temperature-Dependent Spin-Flop in Synthetic Hematite Nanoparticles. Current Nanoscience 2020, 16, 967-975.

19. Greenwood, N.N. Mössbauer Spectroscopy; Springer Netherlands, 1971; ISBN 978-94-009-5699-5.

20. Aljuraide, N.I.; Mousa, M.A.A.; Hessien, M.; Qhatani, M.; Ashour, A.; Wamocha, H.; Hamdeh, H.; Ahmed, M. Structural Properties of Ferric Pseudobrookite $\mathrm{Fe}_{2} \mathrm{Ti}_{1} \mathrm{O}_{5}$ Powder Prepared by a New Method. International Journal of Nanoparticles 2011, 4, 2-9.

21. Hamdeh, H.H.; Xia, Z.; Foehrweiser, R.; McCormick, B.J.; Willey, R.J.; Busca, G. Mössbauer Spectrometry Study of Magnesioferrite Particles. Journal of Applied Physics 1994, 76, 1135-1140, https://doi.org/10.1063/1.357835.

22. Oshtrakh, M.I. Mössbauer Spectroscopy: Application in Biomedical Research. Hyperfine Interact 2005, 165, 313-320, https://doi.org/10.1007/s10751-006-9284-7.

23. Dyar, M.D. Moessbauer Spectra of Biotite from Metapelites. American Mineralogist 1990, 75, 656-666.

24. Tadić, M.; Marković, D.; Spasojević, V.; Kusigerski, V.; Remškar, M.; Pirnat, J.; Jagličić, Z. Synthesis and Magnetic Properties of Concentrated $\alpha-\mathrm{Fe}_{2} \mathrm{O}_{3}$ Nanoparticles in a Silica Matrix. Journal of Alloys and Compounds 2007, 441, 291-296, https://doi.org/10.1016/j.jallcom.2006.09.099.

25. Tadic, M.; Kusigerski, V.; Markovic, D.; Milosevic, I.; Spasojevic, V. High Concentration of Hematite Nanoparticles in a Silica Matrix: Structural and Magnetic Properties. Journal of Magnetism and Magnetic Materials 2009, 321, 12-16, https://doi.org/10.1016/j.jmmm.2008.07.006.

26. Jia, C.-J.; Sun, L.-D.; Yan, Z.-G.; Pang, Y.-C.; You, L.-P.; Yan, C.-H. Iron Oxide Tube-in-Tube Nanostructures. J. Phys. Chem. C 2007, 111, 13022-13027, https://doi.org/10.1021/jp073837q.

27. Eid, C.; Luneau, D.; Salles, V.; Asmar, R.; Monteil, Y.; Khoury, A.; Brioude, A. Magnetic Properties of Hematite Nanotubes Elaborated by Electrospinning Process. J. Phys. Chem. C 2011, 115, 17643-17646, https://doi.org/10.1021/jp203426j.

28. Murphy, J.C.; Foner, S.; Artman, J.O. Magnetic Anisotropy in Antiferromagnetic Corundum-Type Sesquioxides. Phys. Rev. 1965, 138, A912-A917, https://doi.org/10.1103/PhysRev.138.A912.

29. Bilardello, D.; Banerjee, S.K.; Volk, M.W.R.; Soltis, J.A.; Penn, R.L. Simulation of Natural Iron Oxide Alteration in Soil: Conversion of Synthetic Ferrihydrite to Hematite Without Artificial Dopants, Observed With Magnetic Methods. Geochemistry, Geophysics, Geosystems 2020, 21, e2020GC009037, https://doi.org/10.1029/2020GC009037.

30. Grandjean, F.; Long, G.J. Best Practices and Protocols in Mössbauer Spectroscopy. Chem. Mater. 2021, 33, 3878-3904, https://doi.org/10.1021/acs.chemmater.1c00326.

31. Ghafari, M. Unexpected Effect of Boron on Magnetic Properties and Structure of Amorphous Fe-Rich FeSc Alloys. Hyperfine Interact 2021, 242, 4, https://doi.org/10.1007/s10751-021-01727-5. 